\title{
A New Network-Based Strategy for Predicting the Potential miRNA-mRNA Interactions in Tumorigenesis
}

\author{
Jiwei Xue, Fanfan Xie, Junmei Xu, Yuan Liu, Yu Liang, Zhining Wen, and Menglong Li \\ College of Chemistry, Sichuan University, Chengdu 610064, China \\ Correspondence should be addressed to Zhining Wen; w_zhining@163.com and Menglong Li; liml@scu.edu.cn
}

Received 29 April 2017; Accepted 10 July 2017; Published 2 August 2017

Academic Editor: Brian Wigdahl

Copyright (@) 2017 Jiwei Xue et al. This is an open access article distributed under the Creative Commons Attribution License, which permits unrestricted use, distribution, and reproduction in any medium, provided the original work is properly cited.

\begin{abstract}
MicroRNA (miRNA) plays an important role in the degradation and inhibition of mRNAs and is a kind of essential drug targets for cancer therapy. To facilitate the clinical cancer research, we proposed a network-based strategy to identify the cancer-related miRNAs and to predict their targeted genes based on the gene expression profiles. The strategy was validated by using the data sets of acute myeloid leukemia (AML), breast invasive carcinoma (BRCA), and kidney renal clear cell carcinoma (KIRC). The results showed that in the top 20 miRNAs ranked by their degrees, 90.0\% (18/20), $70.0 \%(14 / 20)$, and $70.0 \%(14 / 20)$ miRNAs were found to be associated with the cancers for AML, BRCA, and KIRC, respectively. The KEGG pathways and GO terms enriched with the genes that were predicted as the targets of the cancer-related miRNAs were significantly associated with the biological processes of cancers. In addition, several genes, which were predicted to be regulated by more than three miRNAs, were identified to be the potential drug targets annotated by using the human protein atlas database. Our results demonstrated that the proposed strategy can be helpful for predicting the miRNA-mRNA interactions in tumorigenesis and identifying the cancer-related miRNAs as the potential drug targets.
\end{abstract}

\section{Introduction}

MicroRNAs (miRNAs) are a class of endogenous small noncoding RNA molecule with a length of $\sim 22$ nucleotides, which regulate gene expression posttranscriptionally [1]. miRNAs can combine with mRNAs to form the RNAinduced silencing complex (RISC) and degrade the mRNAs or inhibit the translation of the target genes [2]. The "seed sequence" with a length of $2 \sim 8 \mathrm{nt}$ at the $5^{\prime}$ end of the miRNA plays an important role in target recognition by binding to the complementary sequences in the untranslated regions (3'-UTRs) of mRNAs [3]. A single miRNA may have the capability to target multiple mRNAs $[4,5]$ and participates in multiple signaling pathways and biological processes in mammals. It has been reported that miRNAs are involved in numerous cancer-relevant processes such as cell growth, proliferation, apoptosis, migration, and metabolism [6,7]. The aberrant expression of miRNAs is related to different types of diseases and cancers, such as coronary artery disease [8], gastric cancer [9], lung cancer [10], and breast cancer [11].

Based on the increasing number of studies, miRNAs are being explored as the diagnostic and prognostic biomarkers and as the therapeutic targets for cancer treatment [12]. Previous studies revealed that miRNAs mainly acted as the oncogenic targets or tumor suppressors in the gene regulatory networks [13]. Therefore, two miRNA-based therapeutic strategies were proposed to restore or inhibit miRNA function through miRNA mimics and inhibitors (anti-miRs) [14]. As reported, numerous tumor-suppressive miRNAs and oncogenic miRNAs are promising drug candidates for the treatment of cancers and other diseases [15]. Although most of the miRNA-targeted drugs are still in the preclinical trials, antimiR-122, which is a LNA- (locked nucleic acid-) modified antisense inhibitor, has reached phase II trials for treating hepatitis [16] and the mimics of miR-34, which were encapsulated in lipid nanoparticles, have reached phase I clinical trials for the cancer treatment $[17,18]$. Therefore, it 


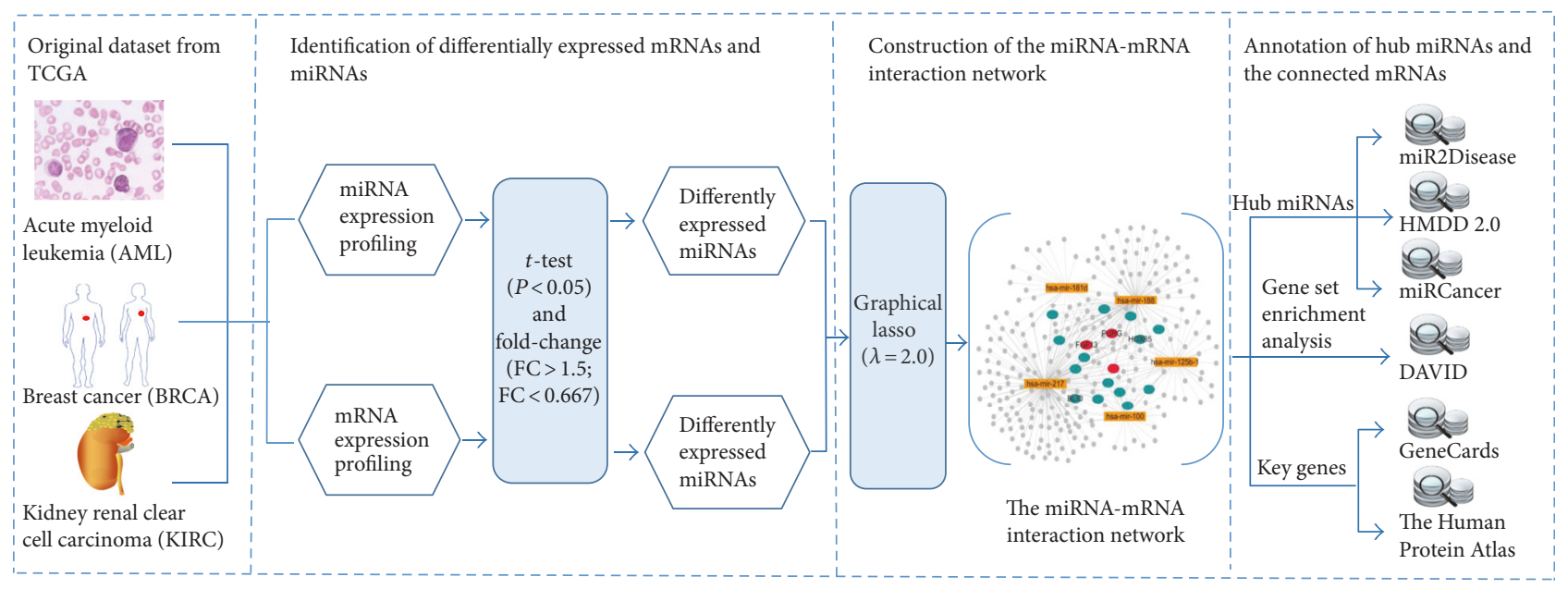

FIGURE 1: The overview of the study design.

TABLE 1: The annotation of the top 20 miRNAs in AML.

\begin{tabular}{|c|c|c|c|}
\hline Cancer type & & Number of genes & Disease \\
\hline \multirow{20}{*}{ AML } & hsa-mir-556 & 170 & - \\
\hline & hsa-mir-217* & 163 & B-cell chronic lymphocytic leukemia, pancreatic neoplasms, nasopharyngeal carcinoma \\
\hline & hsa-mir-636 & 159 & Myelodysplastic syndromes, multiple myeloma \\
\hline & hsa-mir-320c-1 & 147 & Hepatocellular carcinoma, interstitial cystitis \\
\hline & hsa-mir-639 & 145 & Lung cancer, gastric cancer, breast cancer \\
\hline & hsa-mir-873 & 145 & Glioblastoma, endometriosis \\
\hline & hsa-mir-573 & 138 & Pancreatic cancer, esophageal cancer, breast cancer \\
\hline & hsa-mir-216b & 116 & Lung neoplasms, nasopharyngeal neoplasms, colorectal neoplasms \\
\hline & hsa-mir-605 & 109 & Stomach neoplasms, ovarian cancer \\
\hline & hsa-mir-188* & 103 & B-cell chronic lymphocytic leukemia, salivary gland neoplasms, rectal neoplasms \\
\hline & hsa-mir-1468 & 89 & - \\
\hline & hsa-mir-296 & 52 & Glioma, prostate cancer, urinary bladder neoplasms \\
\hline & hsa-mir-488 & 49 & Melanoma, ovarian neoplasms, prostatic neoplasms \\
\hline & hsa-mir-125b-1* & 40 & Acute myeloid leukemia, breast neoplasms, hepatocellular carcinoma \\
\hline & hsa-mir-502 & 36 & Colonic neoplasms, ovarian neoplasms, hepatocellular carcinoma \\
\hline & hsa-mir-551a & 32 & Stomach neoplasms, ovarian cancer \\
\hline & hsa-mir-100* & 30 & $\begin{array}{c}\text { Acute myeloid leukemia, precursor cell lymphoblastic leukemia-lymphoma, } \\
\text { endometrial neoplasms }\end{array}$ \\
\hline & hsa-mir-501 & 29 & Melanoma, atrophic muscular disorders \\
\hline & hsa-mir-520a & 26 & Hodgkin's lymphoma, stomach neoplasms, colorectal neoplasms \\
\hline & hsa-mir-181d* & 25 & Acute myeloid leukemia, acute promyelocytic leukemia, glioblastoma \\
\hline
\end{tabular}

${ }^{*}$ The miRNA was directly associated with AML. ${ }^{-}$No description of the miRNA was found in the disease-related miRNA database.

is essential to identify the key miRNA candidates for the development of miRNA-based therapeutics of the cancers. In recent years, numerous databases, such as miRBase [19], miRanda [20], DIANA-TarBase [21], and HMDD v2.0 [22], have been developed to investigate the key role of $\mathrm{miR}$ NAs in the biological processes and reveal the miRNAmRNA interaction mechanisms. However, considering the fact that a single miRNA will simultaneously target multiple genes, the miRNA-based therapeutics, which were designed to modulate miRNA expression levels, will affect hundreds of genes. It would be harmful for the patient to randomly regulate the hundreds of transcripts [23]. Thus, it is important to provide an exhaustive analysis of the key miRNAs and the miRNA-mRNA interactions before applying the miRNAbased therapeutics to the clinical trials.

In our study, we proposed a strategy by using the graphical lasso algorithm [24] to discover the key miRNAs and the miRNA-mRNA interaction in tumorigenesis based on the 


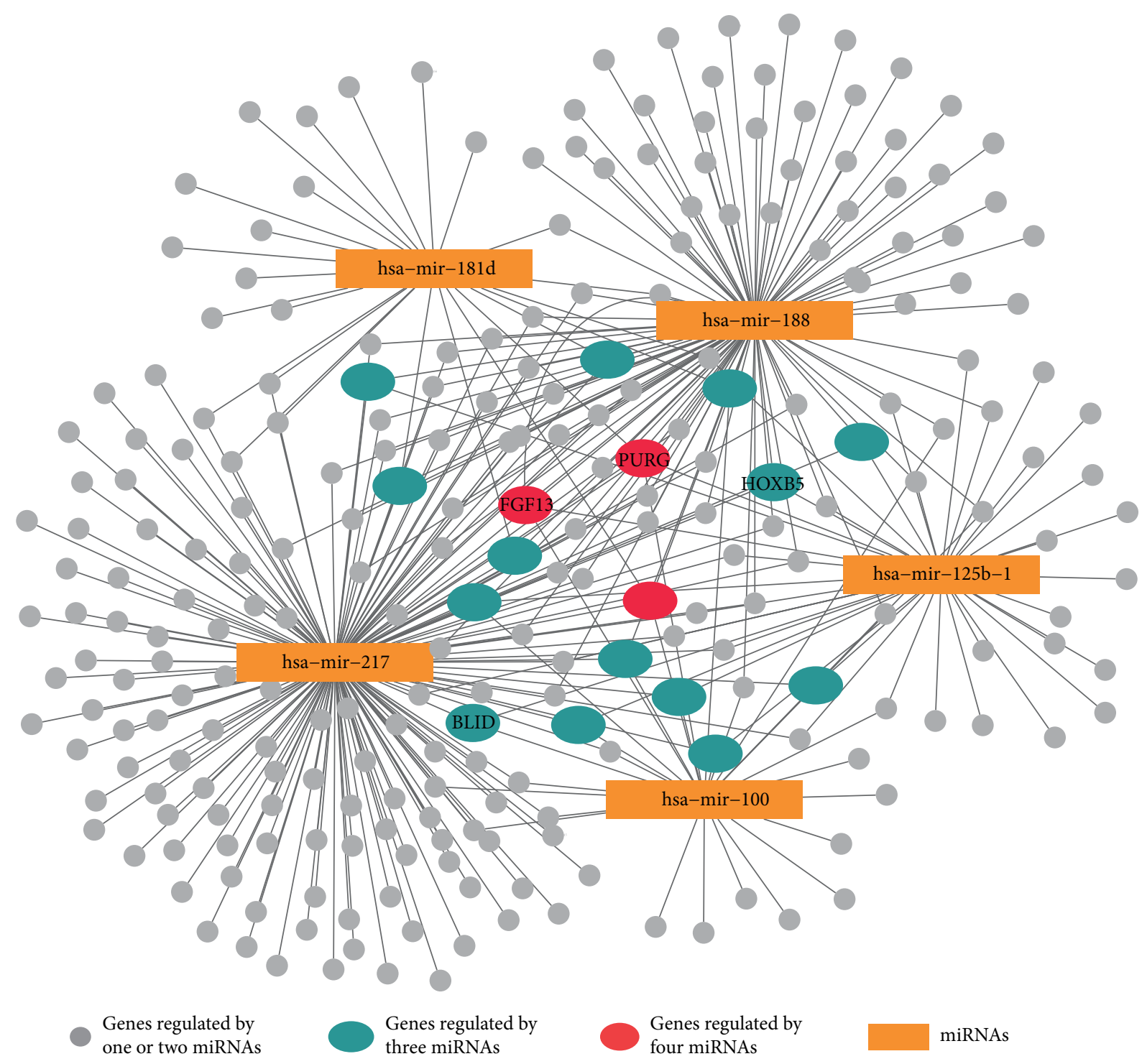

Figure 2: The miRNA-mRNA interaction subnetwork in AML. The five miRNAs in the network were reported to be associated with AML. In the figure, 14 mRNAs (cyan dots) and 3 mRNAs (red dots) were predicted to be connected with three and four miRNAs, respectively. The genes correlated with cancers were marked with their gene symbols.

expression levels of miRNAs and mRNAs. A bipartite network with the miRNAs as hubs was constructed to explore the interactions between the miRNAs and mRNAs, and the top 20 miRNAs ranked by their degrees in the network were verified by using three miRNA disease association databases, namely, miRCancer [25], miR2Disease [26], and HMDD v2.0 [22]. Moreover, the gene set enrichment analysis was conducted for the genes that were predicted as the targets in the network by using Database for the Annotation, Visualization, and Integrated Discovery (DAVID) v6.7 [27]. The proposed strategy was validated by using three cancer data sets. Our results showed that for both three data sets, most of the top 20 miRNAs as well as their targeted genes in the network were highly associated with cancers. In addition, the genes, which were predicted to be regulated by more than three cancer-related miRNAs in our study, had been reported as the potential drug targets in previous studies, indicating the satisfactory performance of our proposed strategy on predicting the cancer-related miRNAs and the interactions between miRNAs and their targeted genes.

\section{Materials and Methods}

2.1. Datasets. The miRNA expression data, the mRNA expression data, and the clinical data of three types of cancers, namely, acute myeloid leukemia (AML) [28], breast invasive carcinoma (BRCA) [29], and kidney renal clear cell carcinoma (KIRC) [30], were downloaded from the Cancer Genome Atlas (TCGA, https://cancergenome.nih.gov/) data portal. The miRNA-seq data in three data sets were generated by an Illumina Genome Analyzer in the Baylor College Human Genome Sequencing Center (BCGSC). The mRNA-seq data of AML (downloaded on November 7,2016 ) were generated by an Illumina Genome Analyzer 
TABLE 2: The annotation of the top 20 miRNAs in BRCA.

\begin{tabular}{|c|c|c|c|}
\hline Cancer type & & Number of genes & Disease \\
\hline \multirow{20}{*}{ BRCA } & hsa-mir-1269 & 381 & Lung cancer, colorectal cancer, hepatocellular carcinoma \\
\hline & hsa-mir-934 & 368 & - \\
\hline & hsa-mir-2115 & 325 & - \\
\hline & hsa-mir-618 & 305 & - \\
\hline & hsa-mir-1251 & 286 & - \\
\hline & hsa-mir-9-3* & 282 & Breast neoplasms, stomach neoplasms, glioblastoma \\
\hline & hsa-mir-105-2 & 268 & Biliary tract neoplasms, hepatocellular carcinoma \\
\hline & hsa-mir-767 & 268 & Melanoma, rhinitis, allergy, perennial \\
\hline & hsa-mir-449a* & 264 & Breast cancer, adenocarcinoma, colonic neoplasms, ovarian neoplasms \\
\hline & hsa-mir-885 & 261 & Leukemia \\
\hline & hsa-mir-105-1 & 253 & Biliary tract neoplasms, hepatocellular carcinoma \\
\hline & hsa-mir-135a- $1^{*}$ & 251 & Breast neoplasms, colorectal neoplasms, non-small-cell lung carcinoma \\
\hline & hsa-mir-3662 & 246 & Gastric cancer, head and neck cancer \\
\hline & hsa-mir-138-1 & 242 & Oral squamous cell carcinoma, renal cell carcinoma, urinary bladder neoplasms \\
\hline & hsa-mir-376a-2 & 234 & Adrenocortical carcinoma, glioblastoma, lung neoplasms \\
\hline & hsa-mir-137* & 233 & Breast neoplasms, malignant melanoma, glioblastoma multiforme \\
\hline & hsa-mir-3190 & 232 & - \\
\hline & hsa-mir-138-2 & 231 & Papillary thyroid carcinoma, oral squamous cell carcinoma, pituitary adenoma \\
\hline & hsa-mir-372 & 231 & Colorectal cancer, acute myeloid leukemia, stomach neoplasms \\
\hline & hsa-mir-3926-2 & 231 & - \\
\hline
\end{tabular}

${ }^{*}$ The miRNA was directly associated with BRCA. ${ }^{-}$No description of the miRNA was found in the disease-related miRNA database.

in the Baylor College Human Genome Sequencing Center (BCGSC). The mRNA-seq data of the BRCA (downloaded on December 15, 2014) and KIRC (downloaded on November 6, 2016) were produced by an Illumina HiSeq 2000 sequencer of the University of North Carolina (UNC). For the three data sets, the read counts for each miRNA and mRNA (data in level 3) were considered the expression level of the miRNA and the mRNA, respectively. In total, we collected 149, 829, and 253 samples for the data sets of AML, BRCA, and KIRC, respectively.

2.2. Study Design. In our study, the graphical lasso algorithm was proposed to construct the miRNA-mRNA interaction network. Figure 1 showed the overview of our study design. Three cancer data sets, namely, AML, BRCA, and KIRC, were downloaded from the TCGA database, and the differentially expressed miRNA and mRNAs were separately identified for each of the data sets by using the fold change ranking combined with a nonstringent $P$ value cutoff. Based on the expression profiles of the differentially expressed miRNAs and mRNAs, the interaction network was constructed by the graphical lasso algorithm, including the connections among the miRNAs and the mRNAs, as well as the connections between miRNAs and mRNAs. The miRNAs and their connected mRNAs in the network were extracted and regrouped into subnetworks, representing the interactions between miRNAs and mRNAs.

To validate whether the cancer-related miRNAs and their key targeted genes can be well characterized by our miRNAmRNA interaction network or not, we annotated the top 20
miRNAs, which were ranked by their degrees (the number of connections), by using three disease-related miRNA databases, namely, miRCancer [25], miR2Disease [26], and HMDD v2.0 [22], for each of the data sets. Meanwhile, the gene set enrichment analysis was conducted with the targeted genes of the cancer-specific miRNAs by using DAVID v6.7. We checked whether or not the significantly enriched Kyoto Encyclopedia of Genes and Genomes (KEGG) pathways and Gene Ontology (GO) terms were associated with cancers. In addition, we mainly discussed the functions of those genes that were predicted as the targets of more than three miRNAs.

2.3. Identification of Differentially Expressed $m R N A s$ and miRNAs. To identify the differentially expressed mRNAs and miRNAs, we firstly divided the samples into two groups for each of the cancer types according to the clinical endpoints. For AML data set, the patients were subdivided into high-risk and low-risk groups according to their survival time. The patients with the survival days longer than one year were assigned to the low-risk group, and the patients with the survival days less than or equal to one year were assigned to the high-risk group. For BRCA data set, the patients were divided into the estrogen receptor- (ER-) positive group and the ER-negative group according to their estrogen receptor status [29]. As to the KIRC data set, the patients in the pathological stages I and II were assigned into the low-risk group and the patients in stages III and IV were assigned into the high-risk group. Then, for all the data sets, Student's $t$-test $P$ value was calculated for each of the miRNAs and mRNAs 


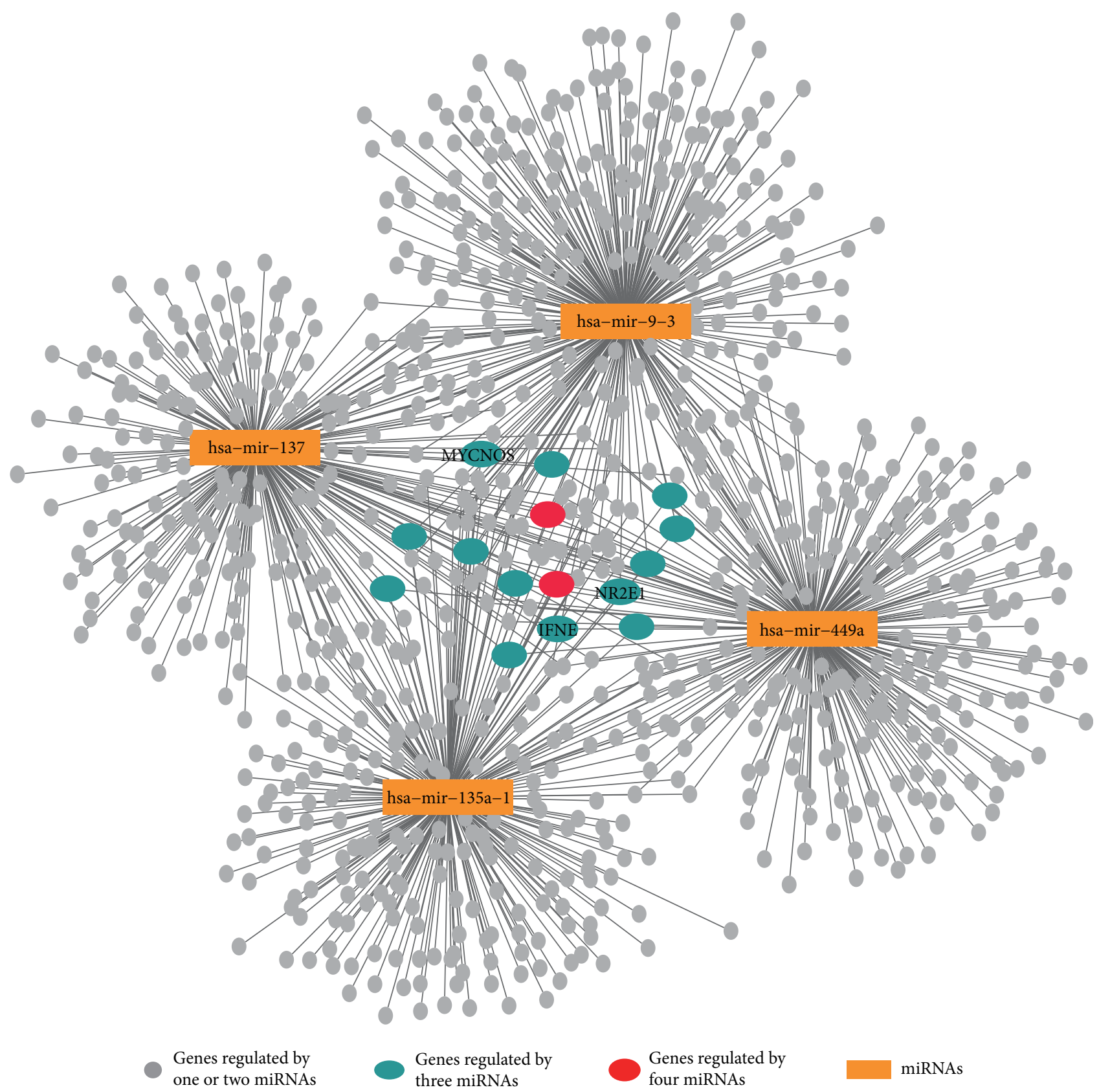

Figure 3: The miRNA-mRNA interaction subnetwork in BRCA. The four miRNAs in the network were reported to be associated with AML. In the figure, 13 mRNAs (cyan dots) and 2 mRNAs (red dots) were predicted to be connected with three and four miRNAs, respectively. The genes correlated with cancers were marked with their gene symbols.

by comparing the expression profiles of the miRNAs and mRNAs between the patient groups. We kept the miRNAs and mRNAs with $P<0.05$ and calculated the fold changes of them between the compared patient groups, respectively. Finally, the miRNAs and the mRNAs with fold change greater than $1.5(\mathrm{FC}>1.5)$ or less than $0.667(\mathrm{FC}<0.667)$ were considered the differentially expressed miRNAs and mRNAs, respectively.

2.4. Construction of the miRNA-mRNA Interaction Network. As reported, Gaussian graphical models (GGMs) have been widely used to identify the dependent relationship among the variables and to be applied on the biological network inference [31, 32]. In GGMs, the conditional dependence of the two nodes was estimated by an inverse covariance matrix. A nonzero number in the inverse covariance matrix indicates a connection between two nodes [33]. The network inference actually is the estimation of the inverse covariance matrix, and numerous algorithms have been proposed to solve this problem [34]. Notably, based on the GGMs, a more reasonable approach named graphical lasso was proposed to directly estimate a sparse inverse covariance matrix by using the L1 (lasso) penalty $[24,35]$. 
TABLE 3: The annotation of the top 20 miRNAs in KIRC.

\begin{tabular}{|c|c|c|}
\hline miRNA & Number of genes & Disease \\
\hline hsa-mir-1291* & 344 & Renal cell carcinoma, ovarian cancer, kidney cancer \\
\hline hsa-mir-558 & 243 & Pancreatic cancer, gastric cancer \\
\hline hsa-mir-3924 & 237 & - \\
\hline hsa-mir-376a-1 & 233 & Salivary gland neoplasms, lung neoplasms, adrenocortical carcinoma \\
\hline hsa-mir-653 & 229 & - \\
\hline hsa-mir-485 & 227 & Ependymoma, non-small-cell lung carcinoma, leukemia \\
\hline hsa-mir-200b* & 216 & Renal cell carcinoma, diabetic nephropathies, pancreatic neoplasms \\
\hline hsa-mir-134* & 215 & Renal cell carcinoma, lupus nephritis, glioblastoma \\
\hline hsa-mir-1246 & 214 & Colorectal neoplasms, esophageal neoplasms \\
\hline hsa-mir-346 & 212 & Lupus nephritis, hepatocellular carcinoma \\
\hline hsa-mir-2110 & 210 & Hepatocellular carcinoma, colorectal neoplasms \\
\hline hsa-mir-365-2 & 210 & - \\
\hline hsa-mir-153-1 & 201 & Endometrial neoplasms, glioblastoma, rectal neoplasms \\
\hline 'hsa-mir-374c & 191 & - \\
\hline hsa-mir-376b & 190 & Adrenocortical carcinoma, uterine leiomyoma, epithelial ovarian cancer \\
\hline hsa-mir-218-2* & 184 & Renal cell carcinoma, lung cancer, urinary bladder neoplasms \\
\hline hsa-mir-300 & 181 & Urinary bladder neoplasms, ovarian neoplasms, heart failure \\
\hline hsa-mir-1303 & 179 & Colorectal neoplasms, hepatocellular carcinoma \\
\hline hsa-mir-676 & 174 & - \\
\hline hsa-mir-1237 & 156 & - \\
\hline
\end{tabular}

${ }^{*}$ The miRNA was directly associated with KIRC. ${ }^{-}$No description of the miRNA was found in the disease-related miRNA database.

We assume a designed $n \times m$ matrix where $n$ indicates the number of samples and $m$ is the number of genes or miRNAs. Let $\boldsymbol{\theta}=\Sigma^{-1}$ and let $\mathbf{S}$ be the empirical covariance matrix; the problem of estimating $\theta$ is converted to maximize the penalized log-likelihood:

$$
\log \operatorname{det} \boldsymbol{\theta}-\operatorname{tr}(\mathrm{S} \boldsymbol{\theta})-\rho\|\boldsymbol{\theta}\|_{1}
$$

where tr indicates the trace. $\|\boldsymbol{\theta}\|_{1}$ is the L1 norm of the matrix, which is the maximum value of the sum of the absolute values of the elements in each of the columns in $\theta$, and $\rho$ is a nonnegative tuning parameter, which controls the sparseness of the network.

In fact, the graphical lasso gets a $\boldsymbol{\theta}_{m \times m}$ matrix to construct the network by using an $n \times m$ matrix as an input. We have two matrices $\mathbf{X}_{n \times j}$ ( $j$ miRNA expression profiles of $n$ samples) and $\mathbf{Y}_{n \times k}(k$ mRNA expression profiles of $n$ samples). Therefore, we integrated these two matrices into the matrix $\mathbf{Z}_{n \times(j+k)}$, which were used to construct an interaction network including the connections among the miRNAs and the mRNAs, as well as the connections between the miRNAs and mRNAs. In our study, only the differentially expressed miRNAs and the mRNAs were used to construct the interaction network and the penalty parameter $\rho$ was set to 2.0 for all the data sets. We mainly concentrated on the interactions between the miRNAs and the mRNA in the network.

\section{Results}

3.1. Most of the Top 20 miRNAs Were Highly Associated with Cancers. For AML data set, 34 differentially expressed
miRNAs and 798 differentially expressed mRNAs were identified from 706 miRNAs and 20,319 mRNAs, respectively. Considering the miRNAs as the hubs of the miRNA-mRNA interaction network, we selected the top 20 miRNAs ranked by their degrees and listed them in Table 1. It can be seen from the table that $90 \%(18 / 20)$ miRNAs were associated with the cancers after being annotated by the three disease-related miRNA databases. Among the cancer-related miRNAs, five miRNAs, namely, hsa-mir-217, hsa-mir-188, hsa-mir-125b1, hsa-mir-100, and hsa-mir-181d, were reported to be associated with the acute myeloid leukemia. Figure 2 showed the subnetworks including these five miRNAs as hubs and their targeted mRNAs.

For the data sets of BRCA and KIRC, we identified 266 and 54 differentially expressed miRNAs from 1043 and 1046 miRNAs, respectively, and identified 6021 and 1647 differentially expressed mRNAs from 20,502 and 20,503 mRNAs, respectively. The top 20 miRNAs ranked by their degrees in the miRNA-mRNA interaction network of the BRCA data set were listed in Table 2. It can be seen that $70 \%(14 / 20)$ miRNAs were annotated to be associated with cancers and four out of them, namely, hsa-mir-9-3, hsamir-449a, hsa-mir-135a-1, and hsa-mir-137, were breast cancer-specific miRNAs. Table 3 showed the top 20 miRNAs that were obtained from the interaction network of KIRC. 14 out of $20(70 \%)$ miRNAs were reported to be associated with cancers, and four out of them, namely, hsa-mir-1291, hsamir-200b, hsa-mir-134, and hsa-mir-218-2 were directly associated with the renal cell carcinoma. The subnetworks 


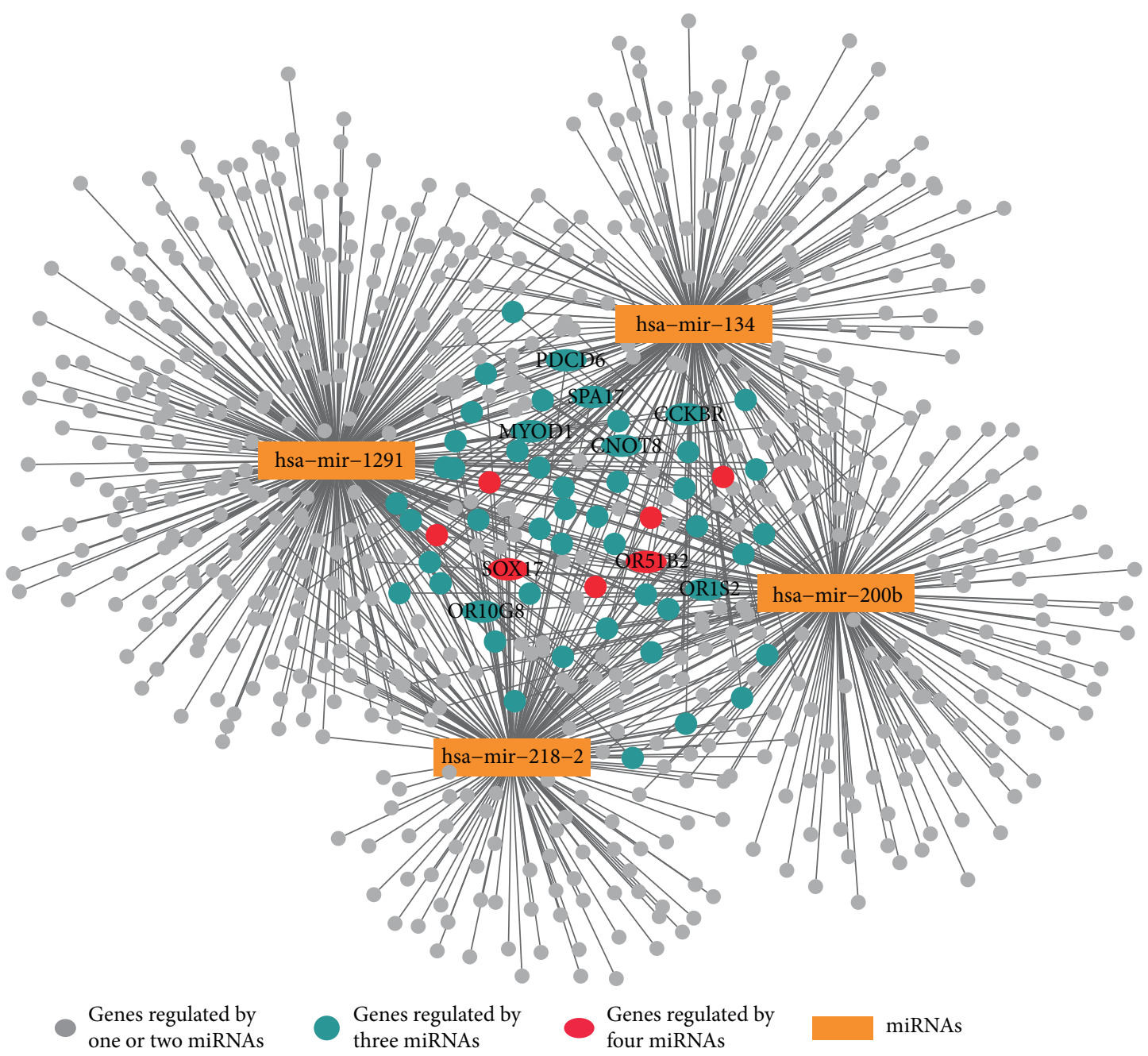

FIgURE 4: The miRNA-mRNA interaction subnetwork in KIRC. The four miRNAs in the network were reported to be associated with AML. In the figure, 49 mRNAs (cyan dots) and 7 mRNAs (red dots) were predicted to be connected with three and four miRNAs, respectively. The genes correlated with cancers were marked with their gene symbols.

TABLE 4: The top 5 KEGG pathways enriched with the genes connected with the cancer-specific miRNAs.

\begin{tabular}{|c|c|c|}
\hline Cancer type & KEGG pathways & $P$ value \\
\hline \multirow{3}{*}{ AML } & hsa00980: metabolism of xenobiotics by cytochrome & 0.0241 \\
\hline & hsa00982: drug metabolism & 0.0263 \\
\hline & hsa04740: olfactory transduction ${ }^{* *}$ & 0.0407 \\
\hline \multirow{5}{*}{ BRCA } & hsa04080: neuroactive ligand-receptor interaction* & $P<0.0001$ \\
\hline & hsa00140: steroid hormone biosynthesis ${ }^{* *}$ & 0.0120 \\
\hline & hsa03320: PPAR signaling pathway** & 0.0176 \\
\hline & hsa04610: complement and coagulation cascades* & 0.0176 \\
\hline & hsa00150: androgen and estrogen metabolism ${ }^{* *}$ & 0.0246 \\
\hline \multirow{5}{*}{ KIRC } & hsa05322: systemic lupus erythematosus & 0.0003 \\
\hline & hsa04060: cytokine-cytokine receptor interaction ${ }^{* *}$ & 0.0021 \\
\hline & hsa04740: olfactory transduction* & 0.0122 \\
\hline & hsa05034: alcoholism & 0.0219 \\
\hline & hsa00350: tyrosine metabolism & 0.0224 \\
\hline
\end{tabular}

**The pathway was directly associated with the corresponding cancer type. ${ }^{*}$ The pathway was associated with other cancers. 
TABLE 5: The top $5 \mathrm{GO}$ terms enriched with the genes connected with the cancer-specific miRNAs.

\begin{tabular}{|c|c|c|c|}
\hline Cancer type & Category & Term & $P$ value \\
\hline \multirow{9}{*}{ AML } & GOTERM_BP_4 & GO:0009887 organ morphogenesis & $P<0.0001$ \\
\hline & & GO:0048705 skeletal system morphogenesis & $P<0.0001$ \\
\hline & & GO:0001501 skeletal system development & $P<0.0001$ \\
\hline & & GO:0003002 regionalization ${ }^{* *}$ & $P<0.0001$ \\
\hline & & GO:0048704 embryonic skeletal system morphogenesis** & $P<0.0001$ \\
\hline & GOTERM_MF_4 & GO:0043565 sequence-specific DNA binding** & 0.0055 \\
\hline & & GO:0003700 transcription factor activity & 0.0075 \\
\hline & & GO:0008236 serine-type peptidase activity & 0.0286 \\
\hline & & GO:0004888 transmembrane receptor activity* & 0.0301 \\
\hline \multirow{10}{*}{ BRCA } & GOTERM_BP_4 & GO:0019226 transmission of nerve impulse & $P<0.0001$ \\
\hline & & GO:0007268 synaptic transmission ${ }^{* *}$ & $P<0.0001$ \\
\hline & & GO:0007417 central nervous system development ${ }^{* *}$ & $P<0.0001$ \\
\hline & & GO:0044057 regulation of system process ${ }^{*}$ & $P<0.0001$ \\
\hline & & GO:0009888 tissue development ${ }^{*}$ & $P<0.0001$ \\
\hline & GOTERM_MF_4 & GO:0030594 neurotransmitter receptor activity** & $P<0.0001$ \\
\hline & & GO:0015267 channel activity* & $P<0.0001$ \\
\hline & & GO:0015075 ion transmembrane transporter activity* & $P<0.0001$ \\
\hline & & GO:0008188 neuropeptide receptor activity** & $P<0.0001$ \\
\hline & & GO:0005179 hormone activity* & $P<0.0001$ \\
\hline \multirow{10}{*}{ KIRC } & GOTERM_BP_4 & GO:0006954 inflammatory response & $P<0.0001$ \\
\hline & & GO:0007186 G protein-coupled receptor signaling pathway** & $P<0.0001$ \\
\hline & & GO:0050707 regulation of cytokine secretion* & $P<0.0001$ \\
\hline & & GO:0050663 cytokine secretion* & 0.0002 \\
\hline & & GO:0050715 positive regulation of cytokine secretion & 0.0003 \\
\hline & GOTERM_MF_4 & GO:0005125 cytokine activity* & 0.0002 \\
\hline & & GO:0004930 G protein-coupled receptor activity** & 0.0002 \\
\hline & & GO:0001664 G protein-coupled receptor binding** & 0.0004 \\
\hline & & GO:0005126 cytokine receptor binding* & 0.0007 \\
\hline & & GO:0004984 olfactory receptor activity* & 0.0013 \\
\hline
\end{tabular}

${ }^{* *}$ The Go term was directly associated with the corresponding cancer type. * The Go term was associated with other cancers.

of the specific cancer-related miRNAs and their targeted mRNAs for the data sets of BRCA and KIRC were shown in Figures 3 and 4, respectively.

3.2. The mRNAs Targeted by the Cancer-Specific miRNAs Were Significantly Associated with the Biological Process of Cancers. The gene set enrichment analysis was conducted to investigate the gene functions by using the mRNAs, which were predicted as the targets of the cancer-specific miRNAs. For the data sets of AML, BRCA, and KIRC, 255, 853, and 670 targeted mRNAs were used for the gene set enrichment analysis, respectively. The top 5 significantly enriched KEGG pathways were listed in Table 4. For the data sets of AML, BRCA, and KIRC, there were one, five, and two signaling pathways, respectively, which were reported to be associated with cancers. Likewise, the top 5 significantly enriched GO terms related to the biological process and the molecular functions were listed in Table 5. There were four, nine, and eight GO terms for the data sets of AML, BRCA, and KIRC, respectively, which were associated with the tumorigenesis of the cancers.

When focusing on the mRNAs that were predicted to be the targets of multiple miRNAs, we found 14, 13, and 49 mRNAs targeted by three miRNAs in the miRNA-mRNA interaction networks of AML, BRCA, and KIRC, respectively. Moreover, three, two, and seven mRNAs were predicted to be targeted by four miRNAs in the networks of AML, BRCA, and KIRC, respectively. Figures 2, 3, and 4 showed the mRNAs targeted by three miRNAs (cyan dots) and four miRNAs (red dots). We also annotated these genes by using the GeneCards database v4.4.2 (http://www.genecards.org/) and found four, three, and nine genes from the networks of AML, BRCA, and KIRC, respectively, which were reported to be associated with cancers. The HUGO gene symbols of the cancer-related genes were marked in Figures 2, 3, and 4. 
TABLE 6: The annotation of the key genes connected with more than three cancer-specific miRNAs in the miRNA-mRNA interaction networks.

\begin{tabular}{|c|c|c|c|}
\hline Cancer type & Gene & Gene description & Protein class \\
\hline AML & ASPG & Asparaginase & Enzymes, predicted intracellular proteins \\
\hline BRCA & AQP2 & Aquaporin 2 (collecting duct) & $\begin{array}{l}\text { Disease-related genes, potential drug targets, predicted } \\
\text { membrane proteins, transporters }\end{array}$ \\
\hline \multirow{6}{*}{ KIRC } & CNOT8 & CCR4-NOT transcription complex subunit 8 & Enzymes, plasma proteins, predicted intracellular proteins \\
\hline & CTPS1 & CTP synthase 1 & $\begin{array}{c}\text { Disease-related genes, enzymes, potential drug targets, } \\
\text { predicted intracellular proteins }\end{array}$ \\
\hline & IFNAR2 & Interferon (alpha, beta, and omega) receptor 2 & $\begin{array}{l}\text { Cancer-related genes, FDA-approved drug targets, } \\
\text { predicted intracellular proteins, predicted membrane proteins }\end{array}$ \\
\hline & MOCS2 & Molybdenum cofactor synthesis 2 & $\begin{array}{c}\text { Disease-related genes, enzymes, potential drug targets, } \\
\text { predicted intracellular proteins }\end{array}$ \\
\hline & PRSS37 & Protease, serine 37 & Enzymes, predicted secreted proteins \\
\hline & $\mathrm{VCP}$ & Valosin-containing protein & $\begin{array}{l}\text { Disease-related genes, enzymes, plasma proteins, potential } \\
\text { drug targets, predicted intracellular proteins, transporters }\end{array}$ \\
\hline
\end{tabular}

\section{Discussion}

In this study, we proposed a new strategy to construct the miRNA-mRNA interaction network based on the expression profiles of miRNAs and mRNAs. The connections between miRNAs and mRNAs were created by the graphical lasso algorithm. We applied the strategy to the three cancer data sets and successfully identified a number of cancer-related miRNAs and their targeted mRNAs.

For the AML data set, $90 \%$ miRNAs in the top $20 \mathrm{miR}$ NAs were found to be associated with cancers (Table 1). Among these miRNAs, hsa-mir-100 was considered a potential tumor-related miRNA, which has been reported to regulate cell differentiation by targeting RBSP3 in acute myeloid leukemia [36]. The pediatric AML patients with the upregulation of miR-100 may have poor relapse-free and overall survival [37]. Moreover, the downregulation of miR-181 family members including miR-181a, miR-181b, miR-181c, and miR-181d was associated with poor prognosis in cytogenetically normal acute myeloid leukemia [38]. For the BRCA data set, $70 \%$ miRNAs in Table 2 were associated with cancers and four of them were specifically associated with the breast cancer. has-miR-9 acted as a tumor suppressor, which can inhibit the proliferation of breast cancer cells [39]. miR-137 is a potential tumor suppressor miRNA, which negatively regulates the gene $E R R \alpha$ (estrogen-related receptor alpha) by targeting the two functional sites in the $3^{\prime}$-UTR of ERR $\alpha$ [40]. As to the KIRC data set, $70 \%$ miRNAs in Table 3 were associated with cancers and four of them had been reported to be associated with the development of the renal cell carcinoma. hsa-mir-134 had been reported as a tumor suppressor and can obstruct the tumor growth and metastasis by inhibiting epithelialmesenchymal transition (EMT) in renal cell carcinoma cells [41]. miR-218 can mediate the focal adhesion pathway and inhibit the cell migration and invasion in renal cell carcinoma [42].

We also inspected the gene functions of the mRNAs, which were predicted to be the targets of the cancer-related miRNAs. The results of gene set enrichment analysis showed that the majority of the KEGG pathways (Table 4) and GO terms (Table 5) were significantly associated with the cancers. In the interaction subnetworks (Figures 2, 3, and 4), several mRNAs targeted by multiple cancer-specific miRNAs were found to have key roles in cancers. For example, the gene SOX17, which was predicted to be regulated by four miRNAs in the subnetwork of KIRC, was considered an important tumor suppressor with aberrant methylation for the cancers $[43,44]$. In addition, the genes targeted by more than three miRNAs in the subnetworks were mapped to the Human Protein Atlas database v16.1 (http://www.proteinatlas.org), and 8 genes were annotated as the potential drug targets (Table 6).

Note that compared to the conventional drug therapies, the miRNA-targeting drugs have been regarded as a highvalue therapy because miRNA may modulate multiple biological processes and pathways. However, there are a lot of challenges for utilizing miRNAs as potential therapeutic targets [45]. The miRNAs regulate tens of thousands of genes which could contribute to both efficacy and unexpected side effects. Therefore, the downstream analysis of genes and pathways regulated by miRNAs should be further elucidated and explored. Due to the complex regulatory mechanisms of the miRNAs, it is still challenging to successfully translate the miRNA-based therapy to the clinics [46]. It is a crucial step in miRNA drug discovery [47] to identify the specific miRNAs as drug targets and clarify the mechanisms of the actions for the key miRNAs. The network-based approach proposed in our study can identify the key miRNAs as well as their targeted mRNAs, which were also significantly associated with the biological process of cancers. It would be helpful for providing the complementary support to the miRNAtargeting drug discovery. In addition, the potential mRNA target could be enriched by integrating the protein structure information and medicinal chemistry. Furthermore, the accumulative information about the side effect and off target relationship from available public resources, such as PharmGKB [48] and the Comparative Toxicogenomics Database (CTD) [49], could be utilized to prioritize the genes regulated by miRNAs for therapeutic target discovery. 


\section{Conclusions}

The network-based strategy proposed in our study can efficiently construct the miRNA-mRNA interaction network in tumorigenesis, which included the important cancer-related miRNAs and their targeted genes. The miRNAs and the targeted genes predicted by using the interaction networks may be considered the potential candidates of drug targets in the cancer research.

\section{Conflicts of Interest}

The authors declare that they have no conflicts of interest.

\section{Acknowledgments}

This work was supported by the grants from the National Natural Science Foundation of China (no. 21575094 and no. 21375090).

\section{References}

[1] Y. Huang, X. J. Shen, Q. Zou, and Q. L. Zhao, "Biological functions of MicroRNAs," Journal of Physiology and Biochemistry, vol. 67, no. 1, pp. 129-139, 2011.

[2] S. H. Chan and L. H. Wang, "Regulation of cancer metastasis by microRNAs," Journal of Biomedical Science, vol. 22, no. 1, p. 9, 2015.

[3] A. E. Pasquinelli, "MicroRNAs and their targets: recognition, regulation and an emerging reciprocal relationship," Nature Reviews Genetics, vol. 13, no. 4, pp. 271-282, 2012.

[4] D. M. Pereira, P. M. Rodrigues, P. M. Borralho, and C. M. Rodrigues, "Delivering the promise of miRNA cancer therapeutics," Drug Discovery Today, vol. 18, no. 5-6, pp. 282-289, 2013.

[5] K. B. Reddy, "MicroRNA (miRNA) in cancer," Cancer Cell International, vol. 15, no. 1, pp. 1-6, 2015.

[6] R. Garzon, M. Fabbri, A. Cimmino, G. A. Calin, and C. M. Croce, "MicroRNA expression and function in cancer," Trends in Molecular Medicine, vol. 12, no. 12, p. 580, 2006.

[7] M. D. Jansson and A. H. Lund, "MicroRNA and cancer," Molecular Oncology, vol. 6, no. 6, p. 590, 2012.

[8] Y. Jin, C. J. Yang, X. Xu, J. N. Cao, Q. T. Feng, and J. Yang, "MiR-214 regulates the pathogenesis of patients with coronary artery disease by targeting VEGF," Molecular and Cellular Biochemistry, vol. 402, no. 1, p. 111, 2015.

[9] Y. Mi, D. Zhang, W. Jiang et al., "miR-181a-5p promotes the progression of gastric cancer via RASSF6-mediated MAPK signalling activation," Cancer Letters, vol. 389, p. 11, 2016.

[10] C. G. Li, M. F. Pu, C. Z. Li et al., "MicroRNA-1304 suppresses human non-small cell lung cancer cell growth in vitro by targeting heme oxygenase-1," Acta Pharmacologica Sinica, vol. 38, no. 1, pp. 110-119, 2017.

[11] M. V. Iorio, M. Ferracin, C. G. Liu et al., "MicroRNA gene expression deregulation in human breast cancer," Cancer Research, vol. 65, no. 16, pp. 7065-7070, 2005.

[12] M. V. Iorio and C. M. Croce, "MicroRNA dysregulation in cancer: diagnostics, monitoring and therapeutics. A comprehensive review," EMBO Molecular Medicine, vol. 4, no. 3, pp. 143-159, 2012.
[13] G. A. Calin and C. M. Croce, "MicroRNA signatures in human cancers," Nature Reviews Cancer, vol. 6, no. 11, pp. 857-866, 2006.

[14] N. Tyagi, S. Arora, S. K. Deshmukh, S. Singh, S. Marimuthu, and A. P. Singh, "Exploiting nanotechnology for the development of microRNA-based cancer therapeutics," Journal of Biomedical Nanotechnology, vol. 12, no. 1, pp. 28-42, 2016.

[15] R. Rupaimoole and F. J. Slack, "MicroRNA therapeutics: towards a new era for the management of cancer and other diseases," Nature Reviews Drug Discovery, vol. 16, no. 3, pp. 203-222, 2017.

[16] H. L. Janssen, H. W. Reesink, E. J. Lawitz et al., "Treatment of HCV infection by targeting microRNA," New England Journal of Medicine, vol. 368, no. 18, pp. 1685-1694, 2013.

[17] A. G. Bader, "miR-34-a microRNA replacement therapy is headed to the clinic," Frontiers in Genetics, vol. 3, p. 120, 2012.

[18] M. S. Beg, A. J. Brenner, J. Sachdev et al., "Phase I study of MRX34, a liposomal miR-34a mimic, administered twice weekly in patients with advanced solid tumors," Investigational New Drugs, vol. 35, no. 2, pp. 1-9, 2016.

[19] A. Kozomara and S. Griffiths-Jones, "miRBase: annotating high confidence microRNAs using deep sequencing data," Nucleic Acids Research, vol. 42, Database issue, pp. D68-D73, 2014.

[20] B. John, A. J. Enright, A. Aravin, T. Tuschl, C. Sander, and D. S. Marks, "Human microRNA targets," PLoS Biology, vol. 2, no. 11, article e363, 2004.

[21] I. S. Vlachos, M. D. Paraskevopoulou, D. Karagkouni et al., "DIANA-TarBase v7. 0: indexing more than half a million experimentally supported miRNA: mRNA interactions," Nucleic Acids Research, vol. 43, Data issue, pp. D153-D159, 2015.

[22] Y. Li, C. Qiu, J. Tu et al., "HMDD v2. 0: a database for experimentally supported human microRNA and disease associations," Nucleic Acids Research, vol. 42, Database issue, pp. D1070-D1074, 2013.

[23] P. Hydbring and G. Badalian-Very, "Clinical applications of microRNAs," F1000 Research, vol. 2, no. 2, p. 136, 2013.

[24] J. Friedman, T. Hastie, and R. Tibshirani, "Sparse inverse covariance estimation with the graphical lasso," Biostatistics, vol. 9, no. 3, pp. 432-441, 2008.

[25] B. Xie, Q. Ding, H. Han, and D. Wu, "miRCancer: a microRNA-cancer association database constructed by text mining on literature," Bioinformatics, vol. 29, no. 5, pp. 638-644, 2013.

[26] Q. Jiang, Y. Wang, Y. Hao et al., "miR2Disease: a manually curated database for microRNA deregulation in human disease," Nucleic Acids Research, vol. 37, Supplement 1, pp. D98-D104, 2009.

[27] G. Dennis Jr, B. T. Sherman, D. A. Hosack et al., "DAVID: database for annotation, visualization, and integrated discovery," Genome Biology, vol. 4, no. 9, article R60, 2003.

[28] Network TCGA, Cancer Genome Atlas Research Network, T. J. Ley et al., "Genomic and epigenomic landscapes of adult de novo acute myeloid leukemia," New England Journal of Medicine, vol. 368, no. 22, pp. 2059-2074, 2013.

[29] Cancer Genome Atlas Network, D. C. Koboldt, R. S. Fulton et al., "Comprehensive molecular portraits of human breast tumors," Nature, vol. 490, no. 7418, pp. 61-70, 2012.

[30] The Cancer Genome Atlas Research Network, "Comprehensive molecular characterization of clear cell renal cell carcinoma," Nature, vol. 499, no. 7456, pp. 43-49, 2013. 
[31] A. Dobra, C. Hans, B. Jones, J. R. Nevins, G. Yao, and M. West, "Sparse graphical models for exploring gene expression data," Journal of Multivariate Analysis, vol. 90, no. 1, pp. 196-212, 2004

[32] G. Song, L. Han, and K. Xie, "Overlapping decomposition for Gaussian graphical modeling," IEEE Transactions on Knowledge \& Data Engineering, vol. 27, no. 8, pp. 22172230, 2015.

[33] Y. Zuo, Y. Cui, G. Yu, R. Li, and H. W. Ressom, "Incorporating prior biological knowledge for network-based differential gene expression analysis using differentially weighted graphical LASSO," BMC Bioinformatics, vol. 18, no. 1, p. 99, 2017.

[34] X. F. Zhang, L. Ou-Yang, X. M. Zhao, and H. Yan, "Differential network analysis from cross-platform gene expression data," Scientific Reports, vol. 6, article 34112, 2016.

[35] J. Xu, R. Jing, Y. Liu, Y. Dong, Z. Wen, and M. Li, “A new strategy for exploring the hierarchical structure of cancers by adaptively partitioning functional modules from gene expression network," Scientific Reports, vol. 6, article 28720, 2016.

[36] Y. S. Zheng, H. Zhang, X. J. Zhang et al., "MiR-100 regulates cell differentiation and survival by targeting RBSP3, a phosphatase-like tumor suppressor in acute myeloid leukemia," Oncogene, vol. 31, no. 1, p. 80, 2012.

[37] J. Bai, A. Guo, Z. Hong, and W. Kuai, "Upregulation of microRNA-100 predicts poor prognosis in patients with pediatric acute myeloid leukemia," Onco Targets Therapy, vol. 5, pp. 213-219, 2012, default.

[38] Z. Li, H. Huang, Y. Li et al., "Up-regulation of a HOXA-PBX3 homeobox-gene signature following down-regulation of miR-181 is associated with adverse prognosis in patients with cytogenetically abnormal AML," Blood, vol. 119, no. 10, p. 2314, 2012.

[39] S. D. Selcuklu, M. T. Donoghue, K. Rehmet et al., "MicroRNA9 inhibition of cell proliferation and identification of novel miR-9 targets by transcriptome profiling in breast cancer cells," Journal of Biological Chemistry, vol. 287, no. 35, pp. 29516-29528, 2012.

[40] Y. Zhao, Y. Li, G. Lou et al., "MiR-137 targets estrogen-related receptor alpha and impairs the proliferative and migratory capacity of breast cancer cells," PloS One, vol. 7, no. 6, article e39102, 2012.

[41] Y. Liu, M. Zhang, J. Qian et al., "miR-134 functions as a tumor suppressor in cell proliferation and epithelial-to-mesenchymal transition by targeting KRAS in renal cell carcinoma cells," Dna \& Cell Biology, vol. 34, no. 6, pp. 429-436, 2015.

[42] T. Yamasaki, N. Seki, H. Yoshino et al., "MicroRNA-218 inhibits cell migration and invasion in renal cell carcinoma through targeting caveolin-2 involved in focal adhesion pathway," Journal of Urology, vol. 190, no. 3, pp. 1059-1068, 2013.

[43] I. Balgkouranidou, A. Karayiannakis, D. Matthaios et al., "Assessment of SOX17 DNA methylation in cell free DNA from patients with operable gastric cancer. Association with prognostic variables and survival," Clinical Chemistry \& Laboratory Medicine, vol. 51, no. 7, pp. 1505-1510, 2013.

[44] D. Fu, C. Ren, H. Tan et al., "Sox17 promoter methylation in plasma DNA is associated with poor survival and can be used as a prognostic factor in breast cancer," Medicine, vol. 4, no. 3, pp. 143-159, 2012.

[45] M. F. Schmidt, "Drug target miRNAs: chances and challenges," Trends in Biotechnology, vol. 32, no. 11, pp. 578-585, 2014.
[46] J. K. Nagpal, R. Rani, B. Trink, and K. S. Saini, "Targeting miRNAs for drug discovery: a new paradigm," Current Molecular Medicine, vol. 10, no. 5, pp. 503-510, 2011.

[47] S. P. Nana-Sinkam and C. M. Croce, "Clinical applications for microRNAs in cancer," Clinical Pharmacology \& Therapeutics, vol. 93, no. 1, p. 98, 2013.

[48] C. F. Thorn, T. E. Klein, and R. B. Altman, "PharmGKB: the pharmacogenomics knowledge base," Methods in Molecular Biology, vol. 1015, pp. 311-320, 2013.

[49] C. J. Mattingly, G. T. Colby, J. N. Forrest, and J. L. Boyer, "The Comparative Toxicogenomics Database (CTD)," Environmental Health Perspectives, vol. 111, no. 6, pp. 793-795, 2003. 

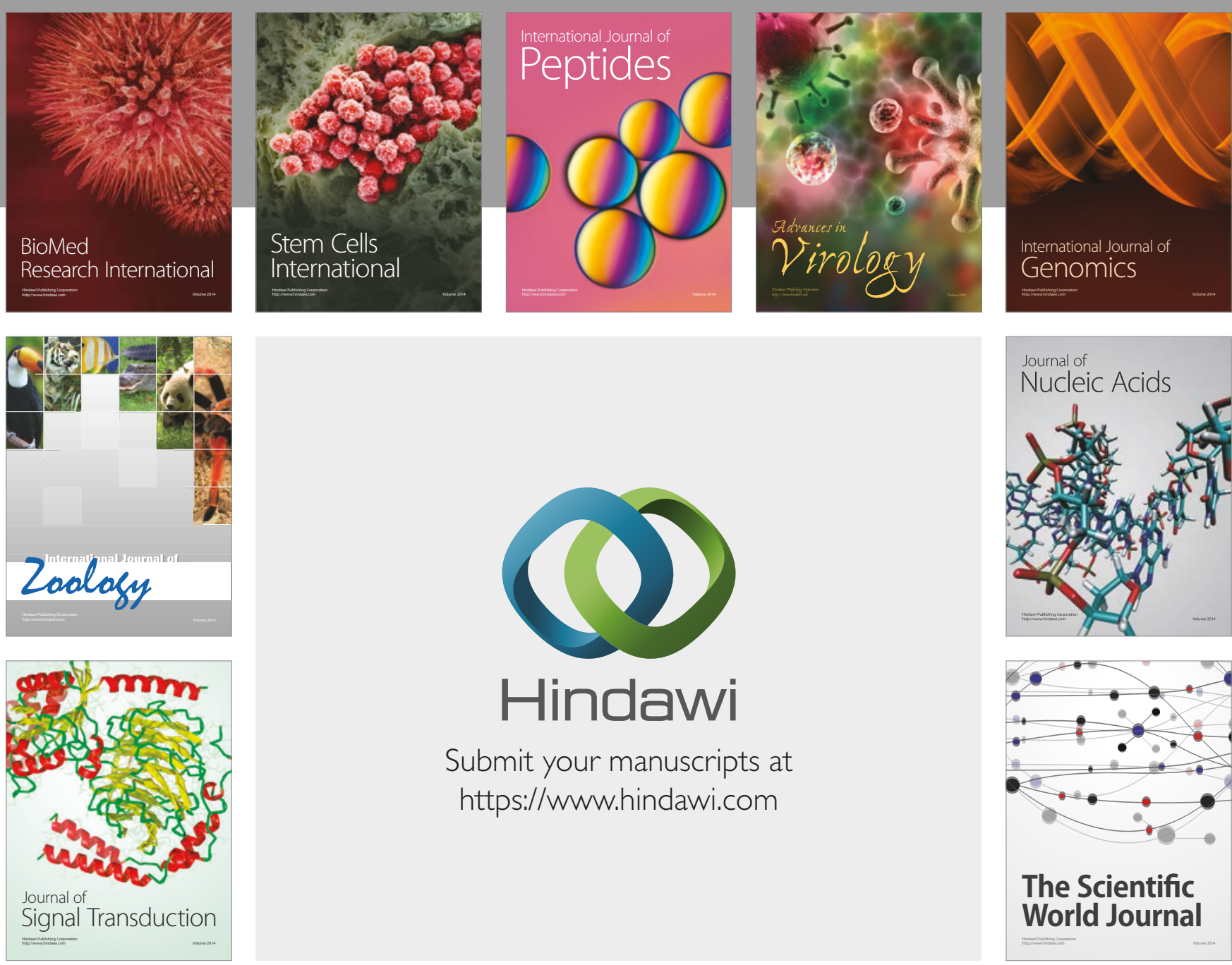

Submit your manuscripts at

https://www.hindawi.com
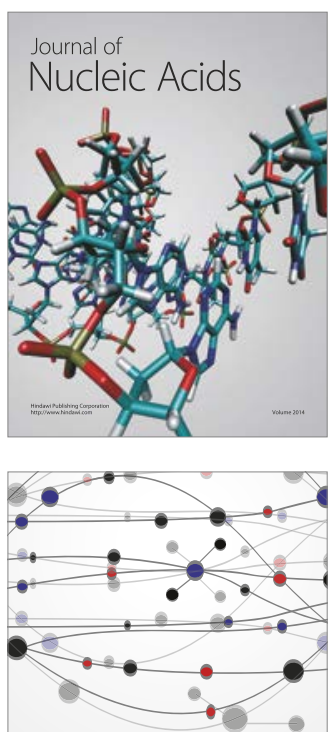

The Scientific World Journal

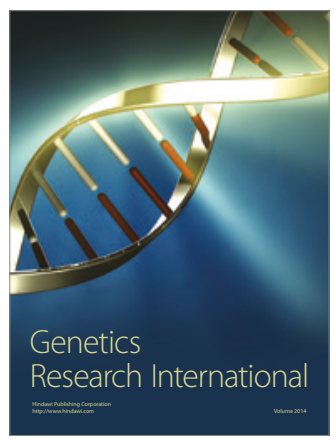

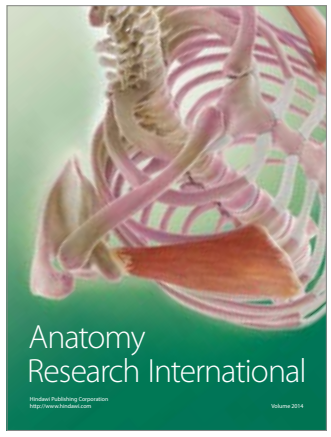

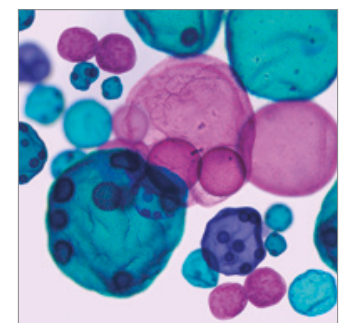

International Journal of Microbiology
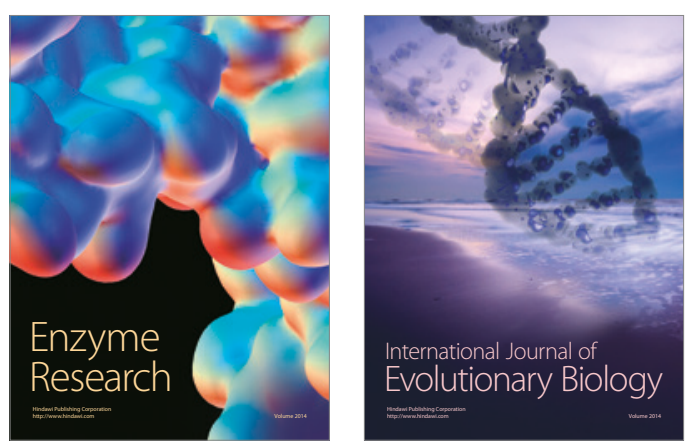
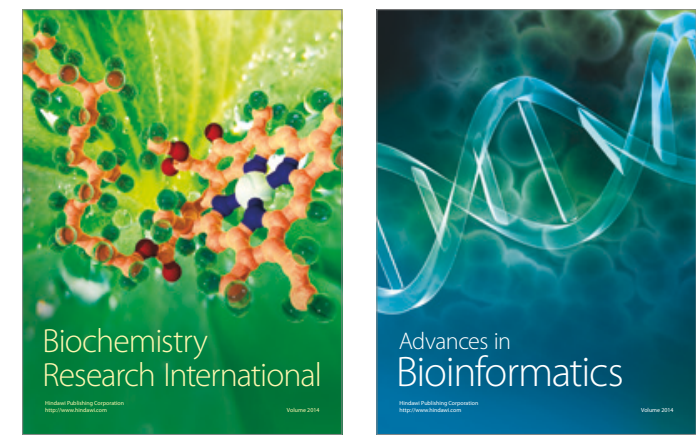

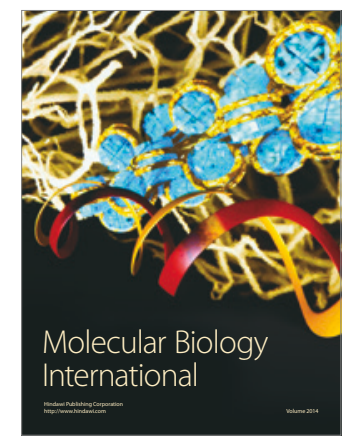

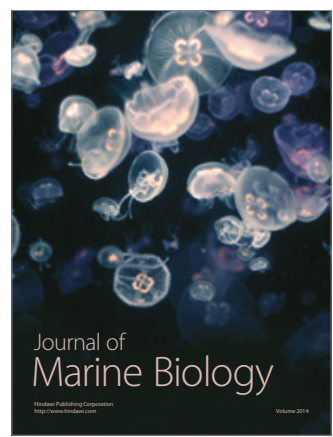

